\title{
Influence of silver ion reduction on electrical modulus parameters of solid polymer electrolyte based on chitosan- silver triflate electrolyte membrane
}

\author{
S. B. Aziz*, Z. H. Z. Abidin, A. K. Arof \\ Center for Ionics, University Malaya, Physics Department, Faculty of Science, 50603 Kuala Lumpur, Malaysia
}

Received 9 January 2010; accepted in revised form 11 March 2010

\begin{abstract}
The electric modulus properties of solid polymer electrolyte based on chitosan: $\mathrm{AgCF}_{3} \mathrm{SO}_{3}$ from 303 to $393 \mathrm{~K}$ have been investigated by using impedance spectroscopy. The shift of the $M^{\prime \prime}$ peak spectra with frequeny depends on the dissociation and association of ions. The lowest conductivity relaxation time $\tau_{\sigma}$, was found for the sample with the highest conductivity. The real part of electrical modulus shows that the material is highly capacitive. The asymmetric peak of the imaginary part of electric modulus $M^{\prime \prime}$, predicts a non Debye type relaxation. The distribution of relaxation times was indicated by a deformed arc form of Argand plot. The increase of $M^{\prime}$ and $M^{\prime \prime}$ values above $358 \mathrm{~K}$ can be attributed to the transformation of silver ions to silver nanoparticles. The complex impedance plots and ultraviolet-visible (UV-vis) absorption spectroscopy indicate the temperature dependent of silver nanoparticles in chitosan-silver triflate solid electrolyte. The formation of silver nanoparticles was confirmed by transmission electron microscopy (TEM). The scaling behavior of $M^{\prime \prime}$ spectra shows that the dynamical relaxation processes is temperature independent for aparticular composition. The $\beta$ exponent value indicate that the conductivity relaxation is highly non exponential.
\end{abstract}

Keywords: polymer membranes, electrical modulus, impedance analysis, UV-vis analysis, transmission electron microscopy

\section{Introduction}

Polymer electrolytes represent a fascinating class of solid-state coordination compounds, which support ionic conductivity in flexible, yet solid membranes [1]. It has been reported that [2] chain polymers, carrying an electro-negative atom (oxygen or nitrogen) in their repeat unit, can act as solvents for certain salts as a consequence of the attractive interaction between chains and cations. Previous studies have proven the existence of both atoms in chitosan polymer [3]. Polymer electrolytes have been studied for their application in batteries, fuel cells, sensors and electrochromic displays [4, 5]. Although these studies revealed many of the properties of these complex systems, the conductivity mechanism is still not fully understood [6]. Usu- ally both crystalline and amorphous phases are present in polymer electrolytes but conductivity mainly occurs in the amorphous phase [7]. The coupling between the polymer segmental relaxation and the ion transport in polymer electrolytes is fascinating; although it is not completely understood, it still holds the key to the development of new energy sources [8].

It has been reported, that the frequency dependent conductivity and relaxation dynamic are both sensitive to the motion of charge species and dipoles of the polymer electrolytes [9]. For close inspection of the relaxation dynamics, the dielectric relaxation can be studied in electric modulus formalism $M^{\prime \prime}$ [10]. This modulus representation has been motivated by $M^{\prime \prime}$ displaying a pronounced peak and 
thereby associating a time scale $\tau$ with the extent of conductivity. On the other hand the modulus representation is still a matter of debate [11], although the relations among the different quantities are well defined. From the physical point of view, the electrical modulus corresponds to the relaxation of the electric field in the material when the electric displacement remains constant [12]. The usefulness of modulus representation is to suppress the signal intensity associated with electrode polarization or to emphasize small features at high frequencies [11]. Thus, the electric modulus spectra provide an opportunity to investigate conductivity and its associated relaxation in ionic conductors and polymers [13].

In this work chitosan has been used as the base polymer for preparation chitosan-silver triflate polymer electrolyte thin films. A monomer of chitosan consists of hydroxyl and amine functional groups which have lone pair electrons that are suitable for the preparation of solid polymer electrolytes [14]. The silver-based polymer electrolytes are prepared by dissolving silver salts such as $\mathrm{AgBF}_{4}, \mathrm{AgClO}_{4}, \mathrm{AgCF}_{3} \mathrm{SO}_{3}$ and $\mathrm{AgSbF}_{6}$ into polymer hosts such as poly(2-ethyl-2-oxazoline) (POZ), poly(ethylene oxide) (PEO) and poly(vinyl pyrrolidone) (PVP) due to coordination interaction between polar groups and silver ions $[15,16]$. The oxygen and nitrogen atoms of polar polymers play an essential role in facilitated olefin transport. However, they also reduce silver ions and create silver metal nanoparticles [17]. This is because silver ions are easily reduced by the lone pair electrons of the nitrogen and oxygen atoms [18]. Each of the nitrogen and oxygen atoms of the functional groups in chitosan has lone pair electrons for occurring complexation as well as reduction of silver ions.

Due to the above reasons the main aim of the present work is to study the influence of silver ion reduction to silver nanoparticle on the electrical modulus properties of chitosan-silver triflate solid electrolyte over a wide range of frequency and temperature.

\section{Experimental}

\subsection{Preparation of SPE thin films}

Chitosan from crab shells ( $\geq 75 \%$ deacetylated, Sigma Aldrich, USA, CAS No. 9012-76-4) and sil- ver triflate $\left(\mathrm{AgCF}_{3} \mathrm{SO}_{3}\right)$ with a molecular weight 256.94 (supplied by Fluka, $\geq 99$ purity, Germany, CAS No. 2923-28-6) have been used as the raw materials in this study. The solid polymer electrolyte (SPE) films were prepared by the solution cast technique with acetic acid (1\%) as solvent. In the present system $1 \mathrm{~g}$ of chitosan was fixed and dissolved in acetic acid solution. To this system silver triflate $\left(\mathrm{AgCF}_{3} \mathrm{SO}_{3}\right)$ was added and varied from 2 to $10 \mathrm{wt} \%$ in steps of $2 \mathrm{wt} \%$ to prepare various composition of chitosan-silver triflate electrolyte in weight percent ratios; 100:0 for pure chitosan acetate, 98:2, 96:4, 94:6, 92:8 and 90:10 for chitosan: $\mathrm{AgCF}_{3} \mathrm{SO}_{3}$. The mixture were stirred continuously until homogeneous solution were obtained. After casting in different Petri dishes, the solutions were left to dry at room temperature for films to form. The films were transferred into a dessicator for continuous drying. This procedure yields mechanically stable and solvent-free films.

\subsection{Complex Impedance measurement}

The complex impedance spectroscopy is used to characterize the electrical properties of the materials. The SPE films were cut into small discs of $2 \mathrm{~cm}$ diameter and sandwiched between two identical circular smooth and cleaned stainless steel electrodes under spring pressure to ensure good electrical contacts between the electrodes and the sample; this enables us to avoid the parasite capacitance induced by the presence of air interstices at the interfaces between the sample and the electrodes. The impedance of the films was measured using the HIOKI $3531 \mathrm{Z}$ Hi-tester (made in Japan, No. 1036555) that was interfaced to a computer in the frequency range from $50 \mathrm{~Hz}$ to $1000 \mathrm{kHz}$. The software controls the measurements and calculates the real and imaginary parts of impedance too. The real $\left(Z^{\prime}\right)$ and imaginary $\left(Z^{\prime \prime}\right)$ part of complex impedance $\left(Z^{*}\right)$ were used for the evaluation of the real $\left(M^{\prime}\right)$ and imaginary $\left(M^{\prime \prime}\right)$ parts of complex electric modulus $\left(M^{*}\right)$ using the Equations (1) and (2) [19]:

$$
Z^{*}=Z^{\prime}-j Z^{\prime \prime}
$$

$$
M^{*}=j \omega C_{0} Z^{*}=M^{\prime}+j M^{\prime \prime}
$$

From Equation (1) and Equation (2) one can get the following Equations (3) and (4): 


$$
\begin{aligned}
& M^{\prime}=\omega C_{0} Z^{\prime \prime} \\
& M^{\prime \prime}=\omega C_{0} Z^{\prime}
\end{aligned}
$$

Here $C_{0}$ is the vacuum capacitance and given by $\varepsilon_{0} A / t$, where $t$ is the thickness and $A$ is the area of the film. $\omega$ is the angular frequency and equal to $\omega=2 \pi f, f$ is the frequency of the applied field.

\subsection{UV-vis and TEM characterization}

The UV-vis spectra of the chitosan-silver triflate electrolyte film have been recorded using a Jasco V-570 UV-Vis-NIR spectrophotometer (Japan, Jasco SLM-468) in the absorbance mode. Transmission electron microscopy (TEM) images of the electrolyte were recorded using a LEO LIBRA (Germany, 120 EFTEM, accelerating voltage $120 \mathrm{kV}$ ) instrument. A drop of the solution of chitosan-silver triflate electrolyte was placed on a carbon coated copper grid and dried at room temperature after removal of excess solution using a filter paper.

\section{Results and discussions}

\subsection{Concentration dependence of $M^{\prime \prime}$ spectra}

Figure 1 shows the frequency dependence of $M^{\prime \prime}$ spectra for different salt concentrations at a fixed temperature. It is clear that the maximum $M^{\prime \prime}$ peak spectra for the highest conducting sample shifts to higher frequency compared to other compositions. The appearance of more than one peaks for pure chitosan and chitosan-silver triflate (98:2) especially at low frequency may be due to polar group relaxations. However, these peaks disappear for the samples with higher salt concentration which

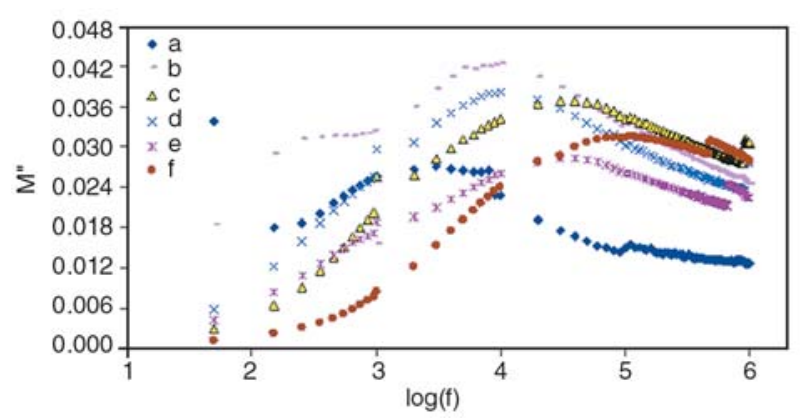

Figure 1. Concentration dependence of $M^{\prime \prime}$ for (a) pure chitosan acetate (100:0), (b) 98:2, (c) 96:4, (d) $94: 6$, (e) $92: 8$ and (f) 90:10 for chitosan: $\mathrm{AgCF}_{3} \mathrm{SO}_{3}$ at $303 \mathrm{~K}$

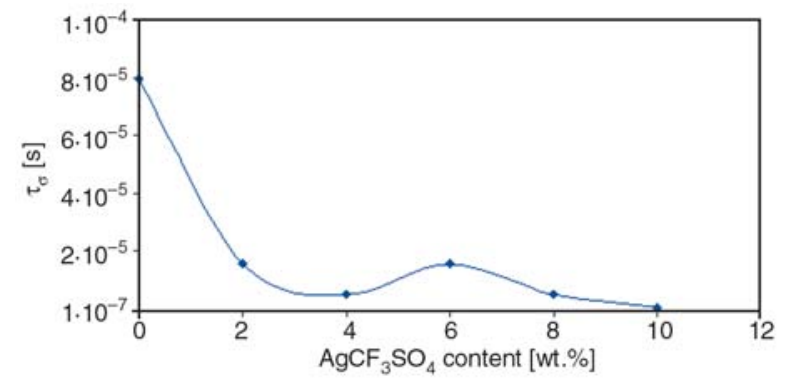

Figure 2. Concentration dependence of conductivity relaxation time $\left(\tau_{\sigma}\right)$ at $303 \mathrm{~K}$

can be ascribed to the large amount of free charge carrier concentration and their motions within the material. Consequently a high DC conductivity is produced which can mask the low frequency relaxation.

These peaks represent the conductivity relaxation peak for the distribution of relaxation times of the free charges and the broadening of the peaks suggests a non-Debye type of relaxation in the material [20]. The peak shifts to higher frequency as the conductivity increases indicating that the relaxation time decreases as conductivity increases [21]. The conductivity relaxation times can be calculated from the relation, $2 \pi f_{\max }=1 / \tau_{\sigma}$, were $\tau_{\sigma}$ is the conductivity relaxation time and $f_{\max }$ is a frequency corresponding to $M_{\max }^{\prime \prime}$. The variation of $\tau_{\sigma}$, as a function of siver triflate concentration is shown in Figure 2. It can be seen that the conductivity relaxation times decrease with increasing salt concentration up to $4 \mathrm{wt} \% \mathrm{AgCF}_{3} \mathrm{SO}_{3}$. From the results it can be inferred that there is competition between ion dissociation and association especially from four to eight weight percentage of salt. The steep decrease in conductivity relaxation time from $8 \mathrm{wt} \%$ $\mathrm{AgCF}_{3} \mathrm{SO}_{3}$ to $10 \mathrm{wt} \% \mathrm{AgCF}_{3} \mathrm{SO}_{3}$ can be explained by an increase in charge carriers concentration, i.e., an increase of conductivity within the sample as a result of increase of a number of mobile charge carriers.

\subsection{Frequency dependence of $M^{\prime}$ and $M^{\prime \prime}$ at selected temperatures}

Figures 3 and 4 show the frequency dependence of real and imaginary parts of complex modulus at several temperatures for the sample with the highest conductivity (90:10). The real and imaginary part of complex modulus were calculated by using Equations (3) and (4). The conductivity behavior in 


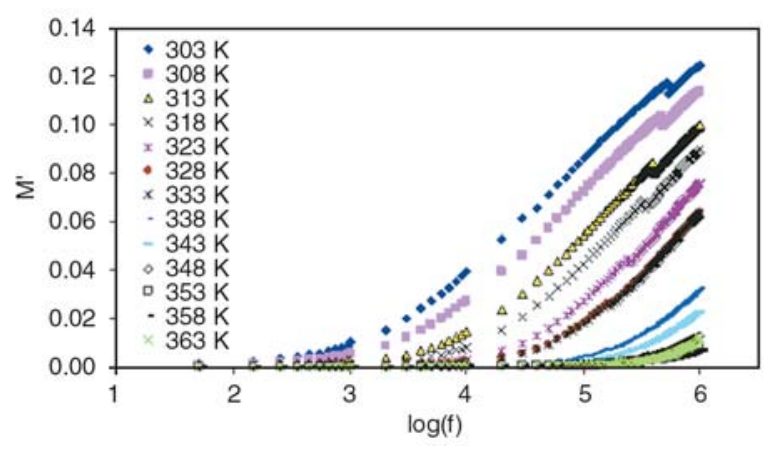

Figure 3. Frequency dependence of $M^{\prime}$ at different temperature for chitosan-silver triflat (90:10)

the frequency domain is more conveniently interpreted in terms of conductivity relaxation time, $\tau_{\sigma}$, using electrical modulus $\left(M^{*}\right)$ representation. The $M^{*}$ representation is now widely used to analyze ionic conductivities by associating a conductivity relaxation time with the ionic process [22]. From Figure 3, it is obvious that at lower frequencies $M^{\prime}$ values are very small, tend to be zero indicating the removal of electrode polarization, $[23,24]$ while the increase of $M^{\prime}$ with increasing frequency and reaching a maximum value $M_{\infty}$ at high frequency, may be due to the distribution of relaxation processes over a range of frequencies [25]. The observed dispersion is mainly due to conductivity relaxation spreading over a range of frequencies and indicates the presence of a relaxation time, which should be accompanied by a loss peak in the diagram of the imaginary part of electric modulus versus frequency. The absence of peak in $M^{\prime}$ diagram is due to the fact that $M^{\prime}$ in complex electric modulus $\left(M^{*}\right)$ is equivalent to $\varepsilon^{\prime}$ in complex permittivity $\left(\varepsilon^{*}\right)$ i.e., $M^{\prime}$ represents the ability of the material to store the energy. The reduction in the values of $M^{\prime}$, at increasing temperature results from the increase in the mobility of the polymer segment and charge carriers with the temperature. It is well

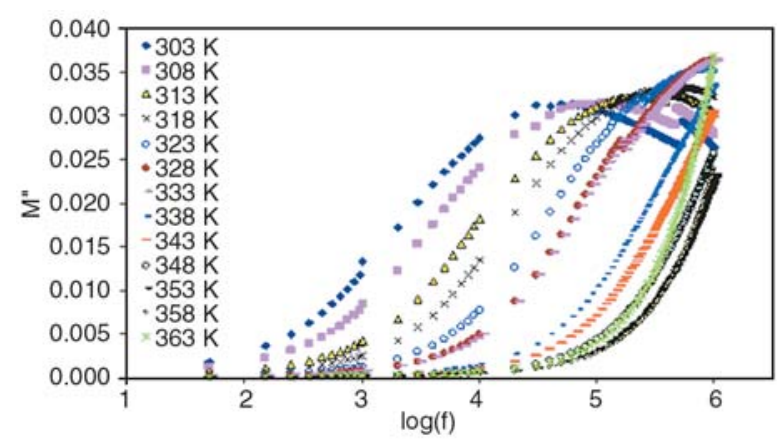

Figure 4. Frequency dependence of $M^{\prime \prime}$ at different temperature for chitosan-silver triflate $(90: 10)$ known that the orientation of the charge carriers and molecular dipoles becomes easier at high temperatures.

The formation of loss peaks is clearly observed in Figure 4. It is obvious that at lower frequencies $M^{\prime \prime}$ exhibits low value, which might be due to the large value of capacitance associated with the electrode polarization effect [26]; as a result of accumulation of a large amount of charge carriers at the electrode/solid polymer electrolyte interface. However, at high frequencies well-defined peaks are observed. The broad and asymmetric of peaks on both sides of the maxima predicts the non-Debye behavior. The region on the left of the peak determines the range in which charge carriers are mobile over long distances, while region to the right is where carriers are confined to potential wells being mobile over short distance [24]. The disappearance of $M^{\prime \prime}$ peak spectra at higher temperatures is due to experimental frequency limitation.

It can be observed that both $M^{\prime}$ and $M^{\prime \prime}$ increase above $358 \mathrm{~K}$, which can be attributed to the reduction of silver ions to silver nanoparticles at these higher temperatures. The reduction of silver ions to silver nanoparticles could be more manifested in the study of $M^{\prime}$ and $M^{\prime \prime}$ as a function of temperature at a fixed frequency.

The frequency associated with each peak is known as relaxation frequency and gives the most probable conductivity relaxation time $\tau_{\sigma}$ for ions. The reciprocal temperature variation of $\log \left(f_{\max }\right)$ is shown in Figure 5. The figure satisfies the Arrhenius behavior with the activation energy, $E_{a}=$ $1.16 \mathrm{eV}$.

The rise in temperature causes the drop in relaxation time due to the increased mobility of ionic carriers. The regression value $R^{2}$ is 0.996 indicating that all points lie on almost straight line.

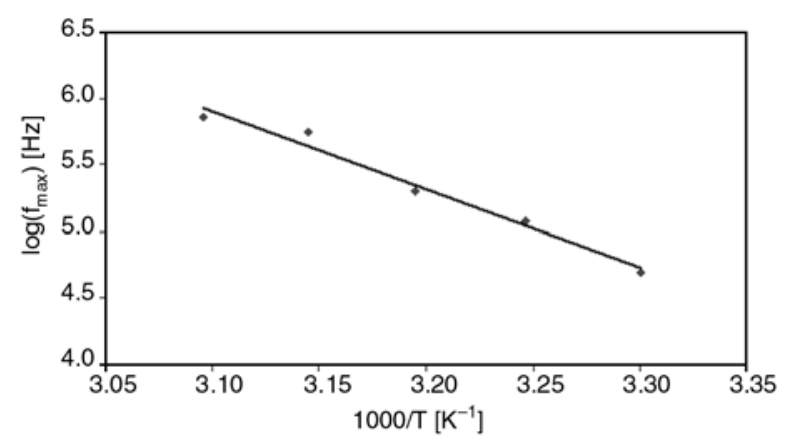

Figure 5. Temperature dependence of relaxation frequency 


\subsection{Argand plots analysis}

The study of Argand plot at different temperatures can be use to demonstrate the nature of relaxation processes in the present polymer electrolyte. Figure 6 shows the temperature dependence of Argand plots.

It is obvious from Figure 6, that the curves of Argand plot are incomplete half semicircle which can not be explained by Debye model (i.e., single relaxation time). In this case a distribution of relaxation time is necessary to interpret the experimental data especially in polymers. Many reasons exist for the relaxation times to be distributed in solids, such as ellipsoidal shape of polar groups, hopping, space charge polarization and the most obvious being the presence of inhomogeneties [27]. Our Argand plots appeared as deformed arcs at low temperatures with their centers positioned below the horizontal axis. This position, in principle, corresponds to the electric relaxation in materials with both the relaxation time and activation energy distributed and intercorrelated [28]. It can be seen (Figure 6) that with increasing temperature the Argand curves shifts towards the origin. This is can be ascribed to the increase of conductivity resulting of increasing of ionic mobility with temperature and thus decreasing of both $Z^{\prime}$ and $Z^{\prime \prime}$. The increase of $M^{\prime \prime}-M^{\prime}$ curve at $363 \mathrm{~K}$ can be attributed to the increase of resistance within the sample due to the reduction of large amount of silver ions to silver nanoparticles.

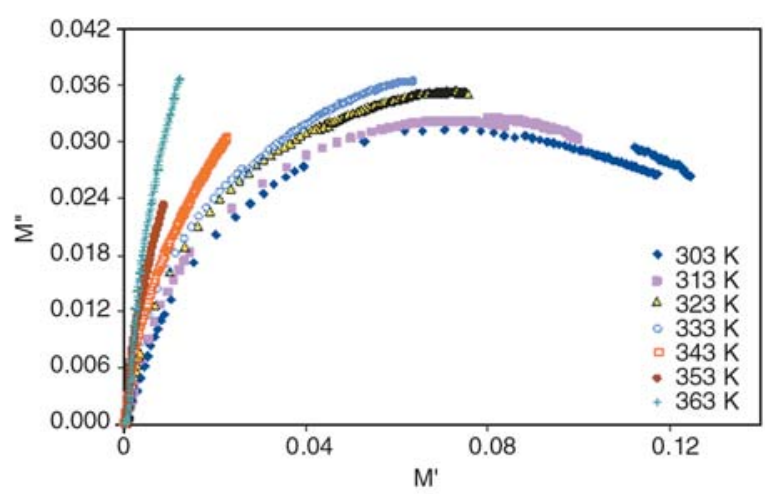

Figure 6. Argand plots for chitosan-silver triflate (90:10) at different temperatures

\subsection{Temperature dependence of $M^{\prime}$ and $M^{\prime \prime}$ at selected frequencies}

Figure $7 \mathrm{a}$ and $7 \mathrm{~b}$ shows the temperature dependence of $M^{\prime}$ and $M^{\prime \prime}$ at selected frequencies. It is
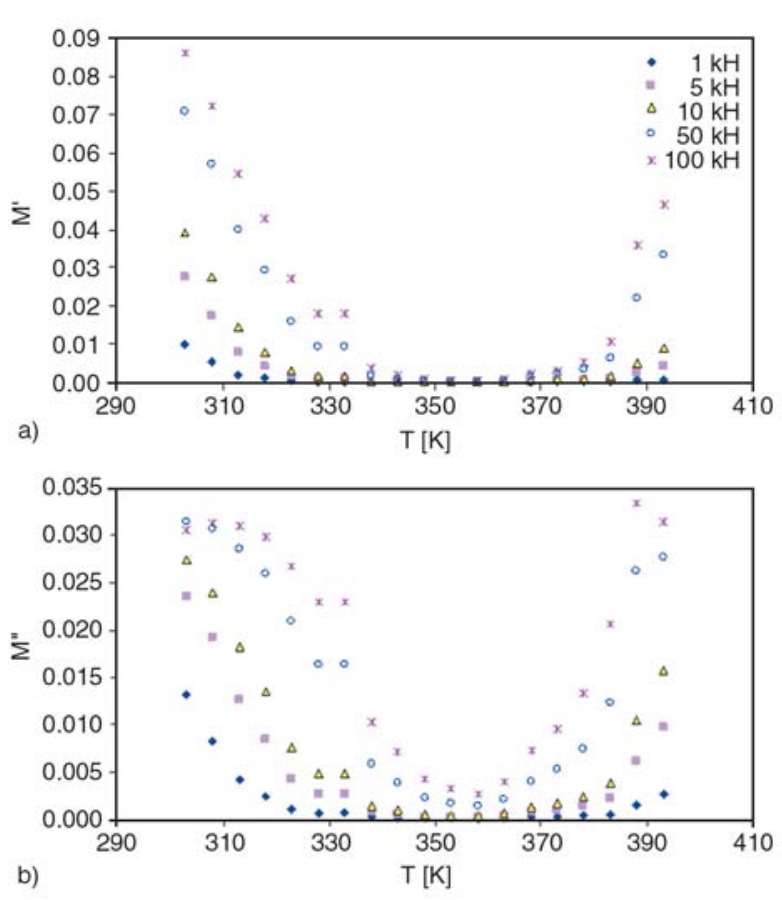

Figure 7. Temperature dependence of (a) $M^{\prime}$ and (b) $M^{\prime \prime}$ at selected frequencies for chitosan-silver triflate (90:10)

clear from the figure that both $M^{\prime}$ and $M^{\prime \prime}$ decrease with increasing of temperature until $358 \mathrm{~K}$, due to the increase of ionic conductivity, i.e., silver ions are dominant. However the increase of $M^{\prime}$ and $M^{\prime \prime}$ can be observed with increasing temperature above $358 \mathrm{~K}$, which can be attributed to the transformation of a large amount of silver ions to silver nanoparticles within the sample. These silver nanoparticles are able to increase the resistance within sample and consequently hinders the ionic motions.

The complex impedance plots can be used as a method to detect the presence of silver nano-particles and their temperature dependence within the present sample. This is due to the powerful ability of electrochemical impedance spectroscopy for the investigation of molecular mobility, phase transitions, conductivity mechanisms and interfacial effects in polymers and complex systems [29].

The complex impedance plots at different temperatures which are shown in Figure 8, are strongly support the above explanation for the increase of $M^{\prime}$ and $M^{\prime \prime}$. The complex impedance plots $\left(Z^{\prime \prime} v s\right.$. $\left.Z^{\prime}\right)$ are commonly used to separate the bulk material (depressed semicircle) and the electrode surface polarization phenomena (tilted spike) [30]. The electrode polarization phenomena (tilted spike) 

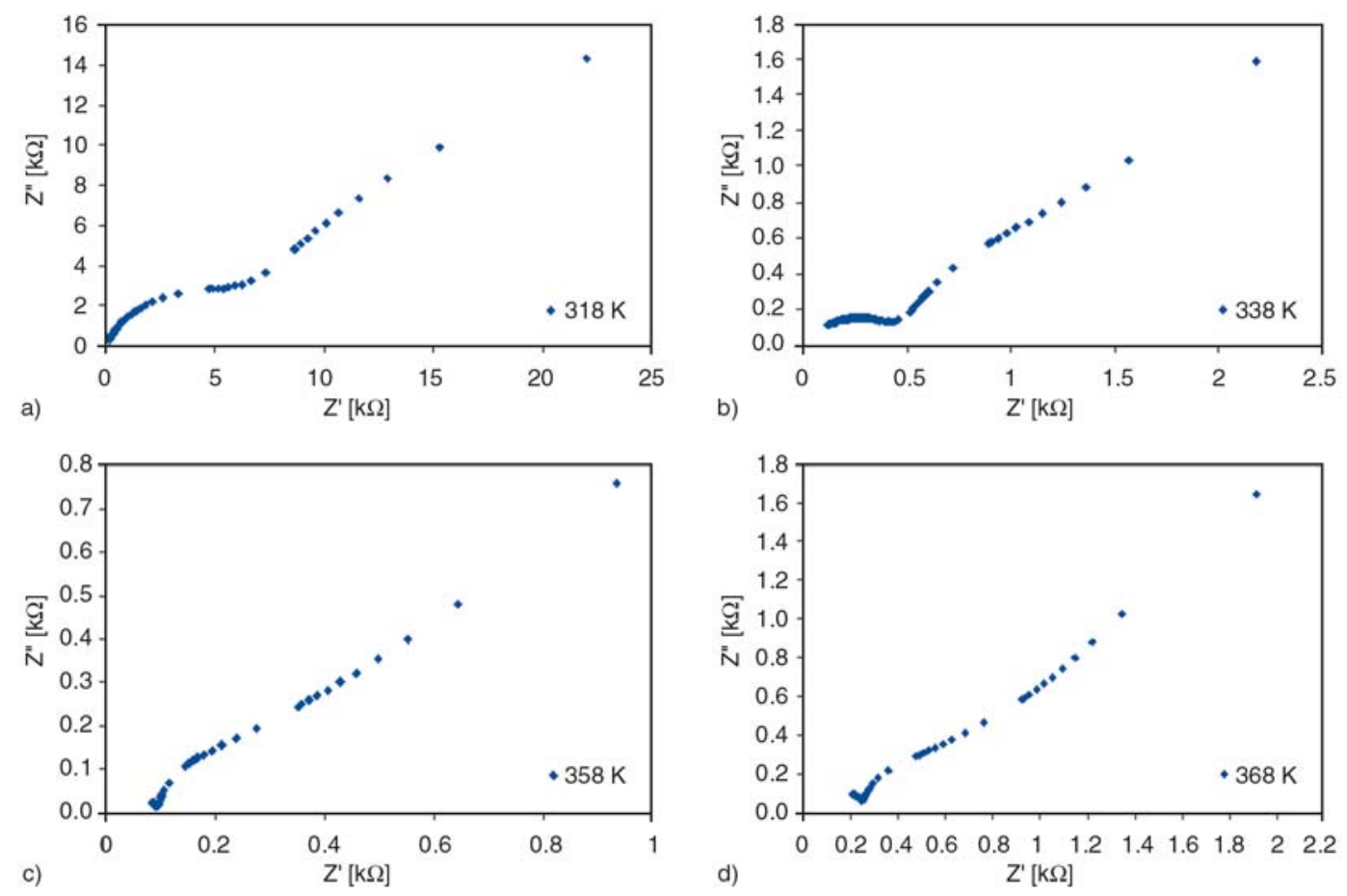

Figure 8. Complex impedance plots of chitosan- $\mathrm{AgCF}_{3} \mathrm{SO}_{3}(90: 10)$ at selected temperatures

occurs due to formation of electric double layer (EDL) capacitances by the free charges build up at the interface between the electrolyte and the electrode surfaces in plane geometry [30, 22]. The appearance of second semicircle at different temperatures can be attributed to silver nanoparticles that acts as grain boundaries in the present system (Figure 8). Thus, there is a competition between silver ions and silver nanoparticles within the same system. It can be noticed that, the bulk resistance decrease with rise in temperature from 303 to $358 \mathrm{~K}$, which indicate the dominance of silver ions, i.e., the system is almost ionic conductor. The increase of bulk resistance after $358 \mathrm{~K}$ demonstrates that with increasing temperature more silver ions converted to silver metal nanoparticles. Thus, transformation from silver ions $\left(\mathrm{Ag}^{+}\right)$to silver nanoparticles $\left(\mathrm{Ag}^{0}\right)$ reduce the number of silver ions that contribute to polarization as well as conduction mechanisms and consequently increase both the $M^{\prime}$ and $M^{\prime \prime}$. This behaviour can be easily deduced from Equation (3) and Equation (4), because above $358 \mathrm{~K}$, both the $Z^{\prime}$ and $Z^{\prime \prime}$ values increases. Consequently both $M^{\prime}$ and $M^{\prime \prime}$ increased. In this case the polymer electrolyte almost exhibits nanocomposite behavior rather than ionic behaviour. However for pure chitosan the second semicircle can not be observed as depicted in Figure 9.
The transformation of silver ion to silver nanoparticle can be understood more obviously when we studying the UV-vis absorption spectra of pure chitosan and chitosan-silver triflate (90:10) solid electrolyte.

Figure 9 shows the complex impedance plots of pure chitosan at different temperatures. It is interesting to note that the bulk resistance of pure chitosan is higher than the bulk resistance of chitosan-silver triflate (90:10) solid electrolyte. In addition the bulk resistance of pure chitosan decrease continiously with increase in temperature untill $393 \mathrm{~K}$, which is completely different as compared to $Z^{\prime \prime}-Z^{\prime}$ plot of chitosan-silver triflate (90:10) solid electrolyte. It is interesting to note that the $Z^{\prime \prime}-Z^{\prime}$ plot of pure chitosan dosen't exhibit any second semicircle at different temperature as manifested in chitosan-silver triflate solid electrolyte.

To investigate the formation of $\mathrm{Ag}$ nanoparticles and their temperature dependence in chitosan-silver triflate system, UV-vis spectroscopy was used as a common characterizing method. This is due to the fact that silver nanoparticles and their clusters exhibit a characteristic UV-vis absorption band in the ultraviolet-visible (UV-vis) region [31]. Figure 10 shows the UV-vis absorption spectra of pure chitosan and chitosan-silver triflate (90:10) solid 

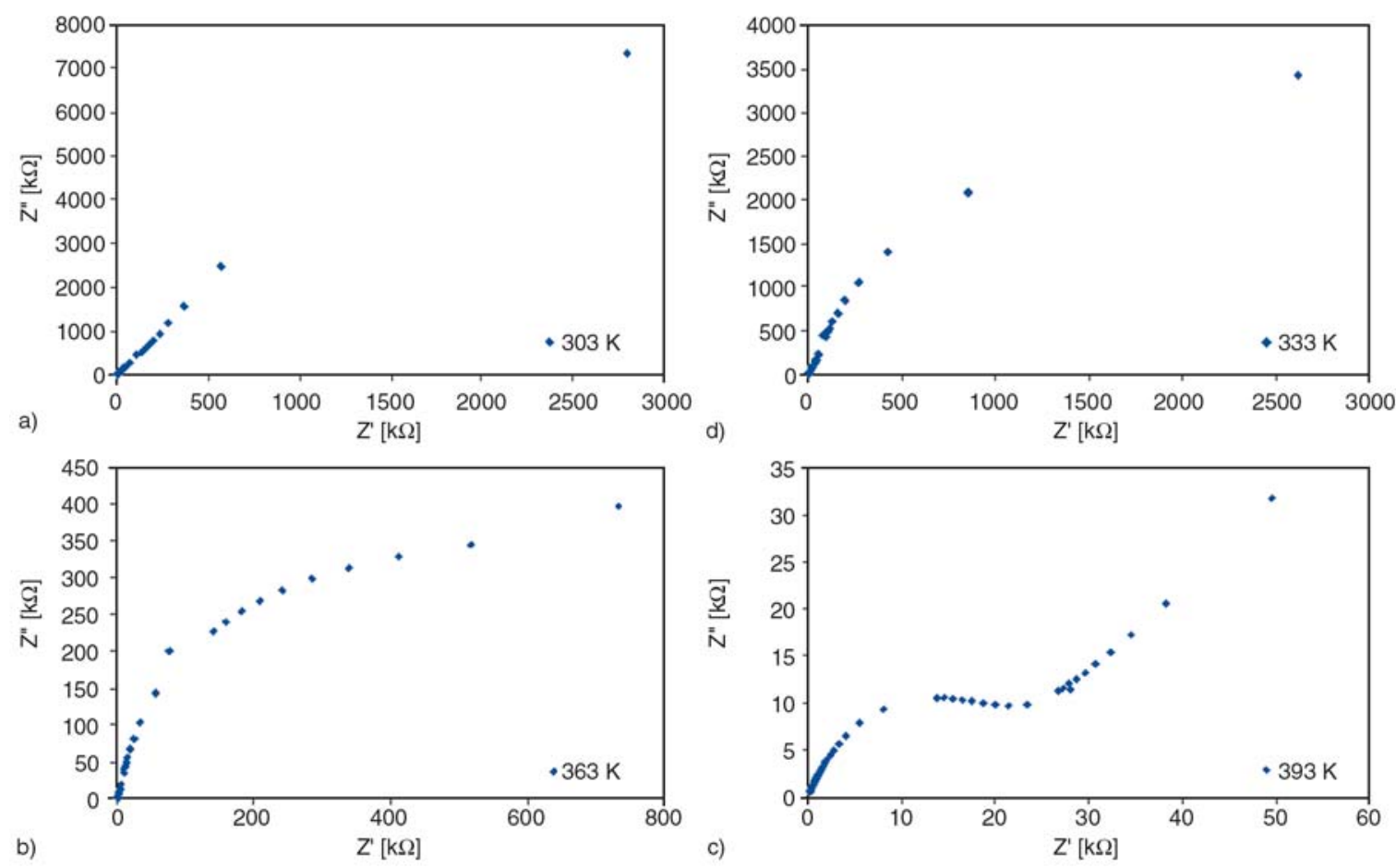

Figure 9. Complex impedance plots of pure chitosan at selected temperatures

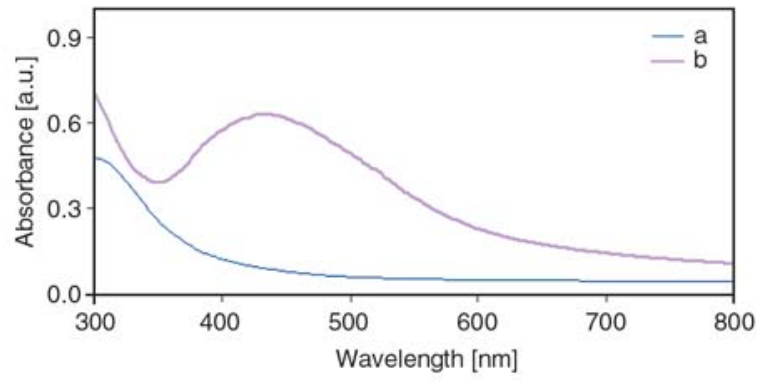

Figure 10. UV-vis absorption spectra of (a) pure chitosan and (b) chitosan- $\mathrm{AgCF}_{3} \mathrm{SO}_{3}$ (90:10) at room temperature $(303 \mathrm{~K})$

electrolyte at ambient temperature (303 K). It can be seen from the figure that pure chitosan has no absorption peak in the region 400 to $500 \mathrm{~nm}$, while a broad absorption peak with a maximum at $426 \mathrm{~nm}$ was observed for chitosan-silver triflate (90:10) solid electrolyte which can be attributed to the surface plasmon band of silver nanoparticles. It is generally accepted that the absorption peak whose maximum occurs at around 420 to $520 \mathrm{~nm}$ is related to the formation of silver metal nanoparticles and its height gives information on the concentration of silver metal nanoparticles [32, 33].

Figure 11 shows the UV-vis absorption spectra for chitosan: $\mathrm{AgCF}_{3} \mathrm{SO}_{3}$ (90:10) solid electrolyte at different temperatures. It can be seen that the height of the peaks increases from 0.61 at $303 \mathrm{~K}$ to 1.2 at $393 \mathrm{~K}$ with increasing temperature, implying an

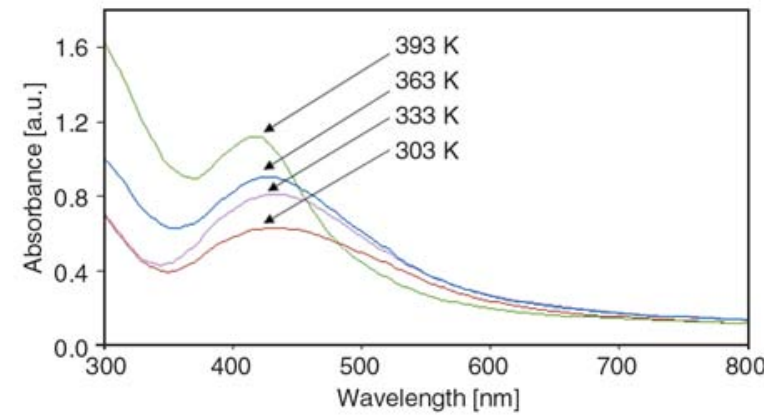

Figure 11. UV-vis spectra of chitosan- $\mathrm{AgCF}_{3} \mathrm{SO}_{3}(90: 10)$ solid electrolyte at different temperatures

increasing amount of Ag nanoparticles because reduction of silver ions to silver nanoparticles occurs more rapidly at a high temperature [34]. It has been reported that polymers which contain hydroxyl, carboxyl, and imide groups are responsible for $\mathrm{Ag}^{+}$ion reduction $[35,36]$. This is due to strong affinity of $\mathrm{N}$ and $\mathrm{O}$ atoms of polar groups for silver ion and metallic silver [36].

The formation of silver nanoparticles in the present system is also indicated by the change in the color of the solid membranes from yellow to dark brown, this phenomenon is also reported by Kang et al. [37] for PVP-silver salt electrolyte. The origin of the intense colour of chitosan-silver triflate is attributed to the surface plasmon resonance (SPR), collective oscillation of free conduction electrons induced by an interacting electromagnetic field 


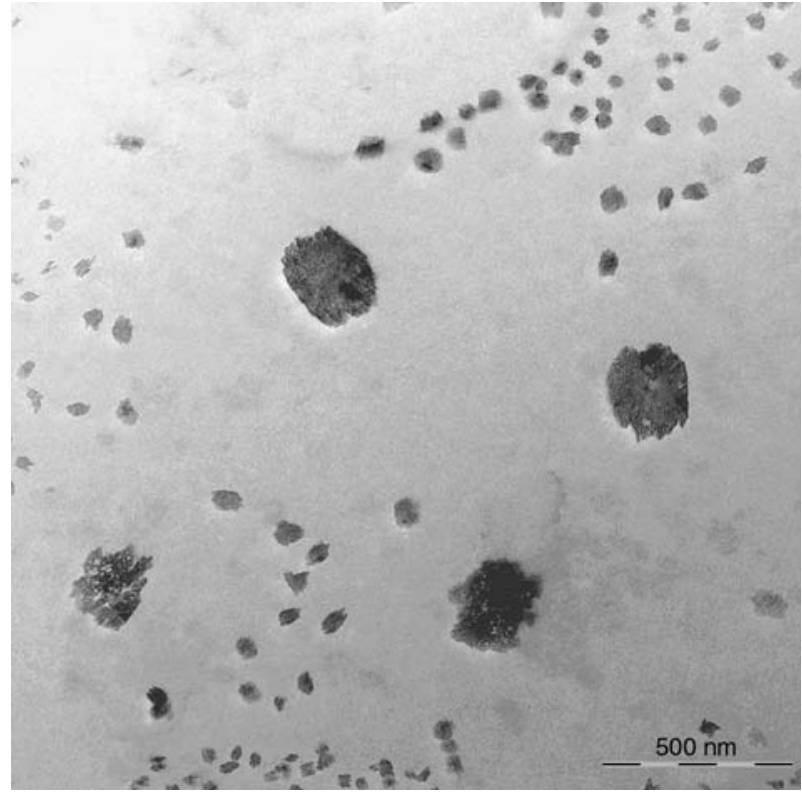

Figure 12. TEM micrograph of silver nanoparticles for chitosan- $\mathrm{AgCF}_{3} \mathrm{SO}_{3}(90: 10)$ at room temperature

[38]. The colour of metal nanoparticles depends on the shape and size of the nanoparticles and dielectric constant of the surrounding medium. However, only electrons with free electron posses plasmon resonance in the visible spectrum, which give rise to such intense color [39].

Transmission electron microscopy (TEM) was used for visual observation of silver nanoparticles in the present system. Figure 12 shows the TEM image of the silver nanoparticles within chtosan-silver triflate (90:10) solid electrolyte. The TEM image indicates that the silver nanoparticles are dispersed and agglomerated. Generally particle agglomeration occurs as a result of larger attraction energy than repulsion energy between the particles [40].

Thus, the increase of $M^{\prime}$ and $M^{\prime \prime}$ at higher temperatures are strongly supported by impedance and UVvis analysis. To our knowledge, this is the first report which discuss the effect of reduction of silver ions to silver nanoparticles on electrical modulus properties of solid polymer electrolyte (SPE).

\subsection{Scaling behavior of $M^{\prime \prime}$}

Scaling of the electric modulus can give further information about the dependence of the relaxation dynamics on the temperature, structure, and also on the concentration of the charge carriers [41]. We have scaled the imaginary part of electric modulus at different temperatures for chitosan: $\mathrm{AgCF}_{3} \mathrm{SO}_{3}$

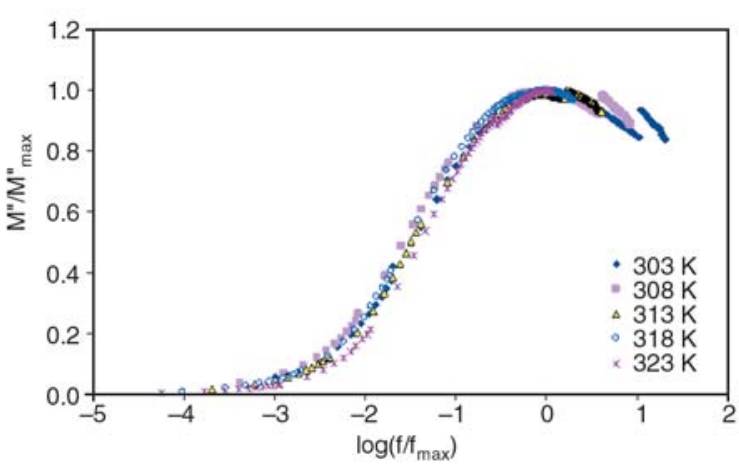

Figure 13. Scaling of $M^{\prime \prime}$ for chitosan- $\mathrm{AgCF}_{3} \mathrm{SO}_{3}(90: 10)$ at different temperatures

(90:10) as shown in Figure 13. The $M^{\prime \prime}{ }_{\max }$ and $f_{\max }$ are used as the scaling parameters for $M^{\prime \prime}$ and $f$ respectively.

It can be seen (Figure 13) that all modulus spectra merge on a single master curve. This indicates that the dynamical relaxation processes occurring at different temperatures are independent of temperature for a particular composition [42]. The asymmetric shape of the plot is a strong evidence that the dielectric relaxation process deviates from the pure Debye behaviour, and a non-symmetric distribution of relaxation times exists [43]. It is obvious from Figure 13, that the normalized modulus plot is non symmetric, in agreement with the non exponential behavior of the electrical function, which is well described by the Kohlrausch-William-Watts exponential function [44], see Equation (5):

$$
\phi=\exp \left[\left(-\frac{t}{\tau}\right)^{\beta}\right] \quad 0<\beta<1
$$

were $\beta$ is an exponent indicating departure from the Debye relaxation. In the present system, the value of full width half height $(F W H H)$ for chitosan: $\mathrm{AgCF}_{3} \mathrm{SO}_{3}(90: 10)$ is about 2 decades, which is greater than 1.14 decades for the ideal Debye behavior. The value of $\beta$ is calculated by Equation (6):

$$
\beta=\frac{1.14}{F W H H}
$$

The small value of $\beta$ (0.57) for the present system indicates that the conductivity relaxation is highly non-exponential [42]. The smaller the value of $\beta$ larger is the deviation of relaxation with respect to Debye type relaxation $(\beta=1)$. The value of $\beta$ for a practical solid electrolyte is clearly less than 1 [20]. 


\section{Conclusions}

Chitosan-silver triflate electrolytes were prepared by solution casting technique. The $M^{\prime \prime}$ spectra shift to higher frequency for the sample with the highest conductivity due to the increase of mobile charge carriers. The long tail of $M^{\prime}$ in the low frequency range indicates the capacitive nature of the system. The broadness of $M^{\prime \prime}$ spectra peaks showing the non Debye type relaxation. The deformed arc shape of Argand plots is due to the distribution of relaxation times. The increase of $M^{\prime}$ and $M^{\prime \prime}$ at elevated temperatures can be ascribed to the reduction of a large amount of silver ions to silver nanoparticles. The appearance of second semicircles in complex impedance plots and their temperature dependence confirms the silver nanoparticles and their growth. The UV/vis spectra show that the number of silver nanoparticles increases with increasing temperature which can be ascribed to the strong interaction of hydroxyl and amine groups of chitosan to silver ions. The formation of silver nanoparticles was confirmed by transmission electron microscopy (TEM). The scaling behavior of spectra shows that the dynamical relaxation processes is temperature independent for aparticular composition. The $\beta$ exponent value indicate that the conductivity relaxation is highly non exponential.

\section{Acknowledgements}

The authors gratefully acknowledge the financial support from University of Malaya in the form of grant (grant No. PS214/2009A) for this research project. Shujahadeen B. Aziz wishes to thank the Kurdistan Regional Government for the scholarship awarded. The authors like to thank Fahmi Fariq for the UV-Vis measurements.

\section{References}

[1] Karan N. K., Pradhan D. K., Thomas R., Natesan B., Katiyar R. S.: Solid polymer electrolytes based on polyethylene oxide and lithium trifluoro- methane sulfonate $\left(\mathrm{PEO}-\mathrm{LiCF}_{3} \mathrm{SO}_{3}\right)$ : Ionic conductivity and dielectric relaxation. Solid State Ionics, 179, 689-696 (2008).

DOI: $10.1016 /$ j.ssi.2008.04.034

[2] Dieterich W., Dürr O., Pendzig P., Bunde A., Nitzan A.: Percolation concepts in solid state ionics. Physica A: Statistical and Theoretical Physics, 266, 229-237 (1999).

DOI: $\underline{10.1016 / S 0378-4371(98) 00597-4}$
[3] Yahya M. Z. A., Arof A. K.: Studies on lithium acetate doped chitosan conducting polymer system. European Polymer Journal, 38, 1191-1197 (2002).

DOI: 10.1016/S0014-3057(01)00290-7

[4] Bhargav P. B., Mohan V. M., Sharma A. K., Rao V. V. R. N.: Investigations on electrical properties of (PVA:NaF) polymer electrolytes for electrochemical cell applications. Current Applied Physics, 9, 165-171 (2009).

DOI: 10.1016/j.cap.2008.01.006

[5] Baskaran R., Selvasekarapandian S., Kuata N., Kawamura J., Hattori T.: Ac impedance, DSC and FT-IR investigations on (x)PVAc- $(1-x) P V d F$ blends with $\mathrm{LiClO}_{4}$. Materials Chemistry and Physics, 98, 55-61 (2006).

DOI: 10.1016/j.matchemphys.2005.08.063

[6] de Jonge J. J., Van Zon A., de Leeuw S. W.: Molecular dynamics study of the influence of the polarizability in $\mathrm{PEO}_{\mathrm{x}} \mathrm{-NaI}$ polymer electrolyte systems. Solid State Ionics, 147, 349-359 (2002).

DOI: 10.1016/S0167-2738(02)00056-5

[7] Avellaned C. O. A., Vieira D. F., Al-Kahlout A., Leite E. R., Pawlicka A., Aegerter M. A.: Solid-state electrochromic devices with $\mathrm{Nb}_{2} \mathrm{O}_{5}$ : Mo thin film and gelatin-based electrolyte. Electrochemica Acta, 53, 16481654 (2007).

DOI: $10.1016 /$ j.electacta.2007.05.065

[8] Natesan B., Karan N. K., Katiyar R. S.: Ion relaxation dynamics and nearly constant loss behavior in polymer electrolyte. Physical Review E, 48, 042801/1042801/4 (2006).

DOI: $10.1103 /$ PhysRevE.74.042801

[9] Singh K. P., Gupta P. N.: Study of dielectric relaxation in polymer electrolytes. European Polymer Journal, 34, 1023-1029 (1998). DOI: $10.1016 / \mathrm{S} 0014-3057(97) 00207-3$

[10] Chabchoub N., Khemakhem H.: Ac ionic conductivity investigations on the $\mathrm{CsK}\left(\mathrm{SO}_{4}\right) \cdot \mathrm{Te}(\mathrm{OH})_{6}$ material. Journal of Alloys and Compounds, 370, 8-17 (2004). DOI: 10.1016/j.jallcom.2003.08.091

[11] Richert R.: The modulus of dielectric and conductive materials and its modification by high electric fields. Journal of Non-Crystalline Solids, 305, 29-39 (2002). DOI: 10.1016/S0022-3093(02)01085-2

[12] Molak A., Paluch M., Pawlus S., Klimontko J., Ujma Z., Gruszka I.: Electric modulus approach to the analysis of electric relaxation in highly conducting $\left(\mathrm{Na}_{0.75} \mathrm{Bi}_{0.25}\right)\left(\mathrm{Mn}_{0.25} \mathrm{Nb}_{0.75}\right) \mathrm{O}_{3}$ ceramics. Journal of Applied Physics D: Applied Physics, 38, 1450-1460 (2005).

DOI: $\underline{10.1088 / 0022-3727 / 38 / 9 / 019}$

[13] Migahed M. D., Ishra M., Fahmy T., Barakat A.: Electric modulus and AC conductivity studies in conducting PPy composite films at low temperature. Journal of Physics and Chemistry of Solids, 65, 1121-1125 (2004).

DOI: $10.1016 /$ j.jpcs.2003.11.039 
[14] Majid S. R., Arof A. K.: Electrical behavior of protonconducting chitosan-phosphoric acid-based electrolytes. Physica B: Condensed Matter, 390, 209-215 (2007).

DOI: $10.1016 /$ j.physb.2006.08.038

[15] Kim J. H., Min B. R., Wong J., Kang Y. S.: Anomalous temperature dependence of facilitated propylene transport in silver polymer electrolyte membranes. Journal of Membrane Science, 227, 197-206 (2003). DOI: $10.1016 /$ j.memsci.2003.08.026

[16] Kang S. W., Kim J. H., Won J., Char K., Kang Y. S.: Effect of amino acids in polymer/silver salt complex membranes on facilitated olefin transport. Journal of Membrane Science, 248, 201-206 (2004).

DOI: $\underline{10.1016 / j . m e m s c i .2004 .08 .028}$

[17] Kim J. H., Wong J., Kang Y. S.: Olefin-induced dissolution of silver salts physically dispersed in inert polymers and their application to olefin/paraffin separation. Journal of Membrane Science, 241, 403-407 (2004).

DOI: $10.1016 /$ j.memsci.2004.05.027

[18] Lim P. Y., Liu R. S., She P. L., Hung C. F., Shih H. C.: Synthesis of Ag nanospheres particles in ethylene glycol by electrochemical-assisted polyol process. Chemical Physics Letters, 420, 304-308 (2006). DOI: $10.1016 /$ j.cplett.2005.12.075

[19] Padmasree K. P., Kanchan D. K.: Modulus studies of $\mathrm{CdI}_{2}-\mathrm{Ag}_{2} \mathrm{O}-\mathrm{V}_{2} \mathrm{O}_{5}-\mathrm{B}_{2} \mathrm{O}_{3}$ system. Materials Science and Engineering B, 122, 24-28 (2005).

DOI: 10.1016/j.mseb.2005.04.011

[20] Ram M., Chakrabarti S.: Dielectric and modulus studies on $\mathrm{LiFe}_{1 / 2} \mathrm{Co}_{1 / 2} \mathrm{VO}_{4}$. Journal of Alloys and Compounds, 462, 214-219 (2008).

DOI: $10.1016 /$ j.jallcom.2007.08.001

[21] Yahya M. Z. A., Arof A. K.: Conductivity and X-ray photoelectron studies on lithium acetate doped chitosan films. Carbohydrate Polymer, 55, 95-100 (2004). DOI: $10.1016 /$ j.carbpol.2003.08.018

[22] Pradhan D. K., Choudhary R. N. P., Samantaray B. K.: Studies of structural, thermal and electrical behavior of polymer nanocomposite electrolytes. Express Polymer Letters, 2, 630-638 (2008).

DOI: $10.3144 /$ expresspolymlett.2008.76

[23] Yakuphanoglu F.: Electrical conductivity and electrical modulus properties of $\alpha, \omega$-dihexylsexithiophene organic semiconductor. Physica B, 393, 139-142 (2007). DOI: $10.1016 /$ j.physb.2006.12.075

[24] Dutta A., Sinha T. P., Jena P., Adak S.: Ac conductivity and dielectric relaxation in ionically conducting soda-lime-silicate glasses. Journal of Non-Crystalline Solids, 354, 3952-3957 (2008). DOI: $\underline{10.1016 / \text { j.jnoncrysol.2008.05.028 }}$

[25] Patro L. N., Hariharan K.: AC conductivity and scaling studies of polycrystalline $\mathrm{SnF}_{2}$. Materials Chemistry and Physics, 116, 81-87 (2009).

DOI: $10.1016 /$ j.matchemphys.2009.02.056
[26] Patro L. N., Hariharan K.: Frequency dependent conduction characteristics of mechanochemically synthesized $\mathrm{NaSn}_{2} \mathrm{~F}_{5}$. Material Science and Engineering: B, 162, 173-178 (2009).

DOI: $\underline{10.1016 / \text { j.mseb.2009.04.003 }}$

[27] Kwan K. C.: Dielectric phenomena in solids. Elsevier, New York. (2004).

[28] Calleja R. D., Matveeva E. S., Parkhutik V. P.: Electric relaxation in chemically synthesized polyaniline: Study using electric modulus formalism. Journal of Non-Crystalline Solids, 180, 260-265 (1995). DOI: $10.1016 / 0022-3093(94) 00470-6$

[29] Psarras G. C., Gatos K. G., Karahaliou P. K., Georga S. N., Krontiras C. A., Karger-Kocsis J.: Relaxation phenomena in rubber/layered silicate nanocomposites. Express Polymer Letters, 1, 837-845 (2007).

DOI: $10.3144 /$ expresspolymlett.2007.116

[30] Sengwa R. J., Choudhary S., Sankhla S.: Low frequency dielectric relaxation processes and ionic conductivity of montmorillonite clay nanoparticles colloidal suspension in poly(vinyl pyrrolidone)-ethylene glycol blends. Express Polymer Letters, 2, 800-809 (2008).

DOI: $10.3144 /$ expresspolymlett.2008.93

[31] Lu J., Suarez J. J. B., Takahashi A., Haruta M., Oyama S. T.: In situ UV-vis studies of the effect of particle size on the epoxidation of ethylene and propylene on supported silver catalysts with molecular oxygen. Journal of Catalysis, 232, 285-295 (2005). DOI: $10.1016 /$ j.jcat.2005.02.013

[32] Kim J. H., Kim C. K., Won J., Kang Y. S.: Role of anions for the reduction behavior of silver ions in polymer/silver salt complex membranes. Journal of Membrane Science, 250, 207-214 (2005).

DOI: $10.1016 /$ j.memsci.2004.10.032

[33] Liu Y., Chen S., Zhong L., Wu G.: Preparation of high-stable silver nanoparticle dispersion by using sodium alginate as a stabilizer under gamma radiation. Radiation Physics and Chemistry, 78, 251-255 (2009). DOI: 10.1016/j.radphyschem.2009.01.003

[34] Kim J. H., Min B. R., Kim H. S., Won J., Kang Y. S.: Facilitated transport of ethylene across polymer membranes containing silver salt: Effect of $\mathrm{HBF}_{4}$ on the photoreduction of silver ions. Journal of Membrane Science, 212, 283-288 (2003). DOI: $10.1016 / \mathrm{S} 0376-7388(02) 00451-9$

[35] Sharma V. K., Yngard R. A., Lin Y.: Silver nanoparticles: Green synthesis and their antimicrobial activities. Advances in Colloid and Interface Science, 145, 83-96 (2009).

DOI: $\underline{10.1016 / j . c i s .2008 .09 .002}$

[36] Silvert P-Y., Herrera-Urbina R., Duvauchelle N., Vijayakrishnan V., Elhsissen K. T.: Preparation of colloidal silver dispersions by the polyol process. Part 1 - Synthesis and characterization. Journal of Materials Chemistry, 7, 293-299 (1997). DOI: $\underline{10.1039 / J M 9960600573}$ 
[37] Kang S. W., Kim J. H., Oh K., Won S. J., Char K., Kim H. S., Kang Y. S.: Highly stabilized silver polymer electrolytes and their application to facilitated olefin transport membranes. Journal of Membrane Science, 236, 163-169 (2004).

DOI: $\underline{10.1016 / \mathrm{j} . \mathrm{memsci} .2004 .02 .020}$

[38] Srivastava S., Haridas M., Basu J. K.: Optical properties of polymer nanocomposites. Bulletin Materials Science, 31, 213-217 (2008).

[39] Zielińska A., Skwarek E., Zaleska A., Gazda M., Hupka J.: Preparation of silver nanoparticles with controlled particle size. Procedia Chemistry, 1, 1560-1566 (2009).

DOI: $10.1016 /$ j.proche.2009.11.004

[40] Lin H-W., Hwu W-H., Ger M-D.: The dispersion of silver nanoparticles with physical dispersal procedures. Journal of Materials Processing Technology, 206, 56-61 (2008).

DOI: 10.1016/j.jmatprotec.2007.12.025
[41] Karmakar A., Majumdar S., Giri S.: Polaron relaxation and hopping conductivity in $\mathrm{LaMn}_{1-\mathrm{x}} \mathrm{Fe}_{\mathrm{x}} \mathrm{O}_{3}$. Physical Review B, 79, 094406/1-094406/7 (2009). DOI: 10.1103/PhysRevB.79.094406

[42] Bhattacharya S., Gosh A.: Relaxation of silver ions in fast ion conducting molybdate glasses. Solid State Ionics, 176, 1243-1247 (2005). DOI: $10.1016 /$ j.ssi.2005.03.002

[43] Psarras G. C., Manolakaki E., Tsangaris G. M.: Dielectric dispersion and ac conductivity in-Iron particles loaded-polymer composites. Composites Part A: Applied Science and Manufacturing, 34, 1187-1198 (2003). DOI: 10.1016/j.compositesa.2003.08.002

[44] Bhattacharya S., Gosh A.: Conductivity relaxation in some fast ion-conducting $\mathrm{AgI}-\mathrm{Ag}_{2} \mathrm{O}-\mathrm{V}_{2} \mathrm{O}_{5}$ glasses. Solid State Ionics, 161, 61-65 (2003). DOI: $\underline{10.1016 / \mathrm{S} 0167-2738(03) 00277-7}$ 